\title{
EEG Frontal Alpha Asymmetry and Dream Affect: Alpha Oscillations over the Right Frontal Cortex during REM Sleep and Presleep Wakefulness Predict Anger in REM Sleep Dreams
}

\author{
(DPilleriin Sikka, ${ }^{1,2}$ Antti Revonsuo, ${ }^{1,2}$ ๑Valdas Noreika, ${ }^{3 *}$ and $\odot$ Katja Valli ${ }^{1,2 *}$ \\ ${ }^{1}$ Department of Psychology and Turku Brain and Mind Center, University of Turku, 20014 Turku, Finland, ${ }^{2}$ Department of Cognitive Neuroscience and \\ Philosophy, University of Skövde, 54128 Skövde, Sweden, and ${ }^{3}$ Department of Psychology, University of Cambridge, Cambridge CB2 3EB, United Kingdom
}

\begin{abstract}
Affective experiences are central not only to our waking life but also to rapid eye movement (REM) sleep dreams. Despite our increasing understanding of the neural correlates of dreaming, we know little about the neural correlates of dream affect. Frontal alpha asymmetry (FAA) is considered a marker of affective states and traits as well as affect regulation in the waking state. Here, we explored whether FAA during REM sleep and during evening resting wakefulness is related to affective experiences in REM sleep dreams. EEG recordings were obtained from 17 human participants $(7 \mathrm{men}$ ) who spent 2 nights in the sleep laboratory. Participants were awakened 5 min after the onset of every REM stage after which they provided a dream report and rated their dream affect. Two-minute preawakening EEG segments were analyzed. Additionally, 8 min of evening presleep and morning postsleep EEG were recorded during resting wakefulness. Mean spectral power in the alpha band $(8-13 \mathrm{~Hz})$ and corresponding FAA were calculated over the frontal (F4-F3) sites. Results showed that FAA during REM sleep, and during evening resting wakefulness, predicted ratings of dream anger. This suggests that individuals with greater alpha power in the right frontal hemisphere may be less able to regulate (i.e., inhibit) strong affective states, such as anger, in dreams. Additionally, FAA was positively correlated across wakefulness and REM sleep. Together, these findings imply that FAA may serve as a neural correlate of affect regulation not only in the waking but also in the dreaming state.
\end{abstract}

Key words: anger; dreaming; emotions; frontal alpha asymmetry; REM sleep

Significance Statement

We experience emotions not only during wakefulness but also during dreaming. Despite our increasing understanding of the neural correlates of dreaming, we know little about the neural correlates of dream emotions. Here we used electroencephalography to explore how frontal alpha asymmetry (FAA) — the relative difference in alpha power between the right and left frontal cortical areas that is associated with emotional processing and emotion regulation in wakefulness-is related to dream emotions. We show that individuals with greater FAA (i.e., greater right-sided alpha power) during rapid eye movement sleep, and during evening wakefulness, experience more anger in dreams. FAA may thus reflect the ability to regulate emotions not only in the waking but also in the dreaming state.

\section{Introduction}

We experience affect (i.e., emotions and mood) not only during wakefulness but also during sleep, especially during rapid eye

\footnotetext{
Received Nov. 21, 2018; revised March 12, 2019; accepted March 16, 2019.

Author contributions: P.S., A.R., V.N., and K.V. designed research; P.S. and K.V. performed research; P.S. and V.N. analyzed data; P.S. wrote the paper; A.R., V.N., and K.V. edited the paper.

This work was supported by Academy of Finland Grant 8124623 to A.R., Finnish Cultural Foundation to P.S., Turku University Foundation to P.S., Alfred Kordelin Foundation to P.S., and International Association for the Study of Dreams and Dream Science Foundation Research Grant to P.S., A.R., V.N., and K.V. We thank Tor Lehtonen, Teemu Paldanius, Lotta Sundelin, and Tiina Virta for assistance in data collection; and Dr. Kaili Clackson for comments and suggestions on the manuscript.
}

movement (REM) sleep dreaming (Hobson et al., 2000; Desseilles et al., 2011). While considerable effort has been devoted to understanding the neural basis of dreaming (for review, see Cipolli et al., 2017), little is known about the neural processes underlying dream affect.

The authors declare no competing financial interests.

*V.N. and K.V. contributed equally to this work as last authors.

Correspondence should be addressed to Pilleriin Sikka at pilleriin.sikka@his.se or pilsik@utu.fi.

https://doi.org/10.1523/JNEUROSCI.2884-18.2019

Copyright $\odot 2019$ the authors 
Dreaming refers to the presence of subjective experiences during sleep and is often understood as a simulation of waking life (e.g., Foulkes, 1985; Nielsen, 2010; Hobson and Friston, 2012; Windt, 2015; Revonsuo et al., 2016; Domhoff, 2018). In fact, the phenomenology and the underlying neural processes of dream experiences are argued to overlap with those of waking experiences (De Gennaro et al., 2012; Fox et al., 2013; Wamsley, 2013; Domhoff and Fox, 2015). Studies show that the neural correlates of the specific contents of subjective experience, such as thinking, perceiving, moving, and speaking, are similar in waking and dreaming (Dresler et al., 2011; Horikawa et al., 2013; Perogamvros et al., 2017; Siclari et al., 2017). Therefore, dream affect can also be expected to share neural mechanisms with those of waking affect. Indeed, there is ample evidence for the involvement of affective brain regions during REM sleep (Maquet et al., 1996; Nofzinger et al., 1997; Braun et al., 1998). However, the measurement of brain activity alone is not sufficient to understand the conscious subjective experience an individual is having. If we want to know what people are experiencing, we have to ask them (Barrett, 2004). The study of dreaming is challenging because people cannot report their dream experiences as they are happening but only as they are recalled upon awakening. Studies linking the affective content of dream reports to structural brain differences have yielded inconclusive results (De Gennaro et al., 2011; Blake et al., 2019). Surprisingly, hardly any studies have related affective dream experiences-recalled and reported upon awakening - to the neural processes of preawakening sleep (assumed to occur at the time of having those experiences) (Nielsen and Chénier, 1999; Daoust et al., 2008; Sterpenich et al., 2019). Thus, it is unclear whether and to what extent the neural processes underlying affective experiences are shared across wakefulness and dreaming.

EEG frontal alpha asymmetry (FAA) is considered a marker of affective states and traits as well as of affect regulation in the waking state (for review, see Reznik and Allen, 2018). FAA refers to the difference in alpha power $(8-13 \mathrm{~Hz})$ between the right and left hemisphere over the frontal cortical regions (typically between EEG electrodes F4-F3). Because alpha oscillations have been suggested to reflect inhibition of cortical activity (Klimesch et al., 2007; Jensen and Mazaheri, 2010; Klimesch, 2012) and due to the contralateral inhibitory connections between the hemispheres (Schutter and Harmon-Jones, 2013; Grimshaw and Carmel, 2014), higher FAA scores (i.e., relatively more alpha in the right hemisphere) are assumed to reflect either reduced right or enhanced left frontal activity. Originally, FAA was presumed to reflect affective valence: positive affect related to greater relative left, and negative affect to greater relative right, frontal activity (e.g., Davidson et al., 1979; Tomarken et al., 1992). Many studies have challenged this by showing that both trait and state anger are associated with greater left (or reduced right) frontal activity (Harmon-Jones and Gable, 2018). As a result, FAA is assumed to reflect motivational direction: approach-related affective states and traits (e.g., elation, anger) related to greater left, and withdrawal-related affective states and traits (e.g., fear, anxiety) to greater right, frontal activity (e.g., Davidson, 1998; Reznik and Allen, 2018). However, due to inconsistent results regarding the relationship between right frontal activity and withdrawalrelated affect, it was recently proposed that FAA reflects supervisory control: greater right frontal activity is assumed to be related to enhanced inhibitory control in general and of affective states in particular (Gable et al., 2015, 2018). Indeed, it has been shown that expressing anger is related to relatively greater left, whereas controlling anger is related to relatively greater right, frontal ac- tivity (Hewig et al., 2004). The role of the right PFC in affect regulation is further supported by lesion (e.g., Salas et al., 2016) and neuroimaging (for review, see Ochsner et al., 2012) studies.

Although the relationship between FAA and affect in the waking state is well documented, to the best of our knowledge, only one study has been published on the relationship between REM sleep FAA and dream affect (Daoust et al., 2008). However, in this study, all affective dream experiences (regardless of valence and motivational direction) and participants (healthy and those with autistic spectrum disorders) were pooled together, and only one dream report per participant analyzed, which may explain the null results obtained.

Therefore, our first aim was to investigate the relationship between REM sleep FAA and dream affect. In our previous study (Sikka et al., 2014), we found that in laboratory REM sleep dreams participants rated interest and anger as the most frequently and intensely experienced positive and negative affect, respectively. We focused specifically on these discrete affective states because they can be clearly categorized as high-approach positive affect and high-approach negative affect, respectively. Moreover, inclusion of these two affective states provides a concurrent test of the three theoretical accounts of the purported role of FAA in affective processing. According to the affective valence account (e.g., Tomarken et al., 1992; Harmon-Jones and Gable, 2018), interest should be positively, and anger negatively, associated with FAA. According to the motivational direction account (e.g., Davidson, 1998; Harmon-Jones and Gable, 2018), both interest and anger should be positively related to FAA. According to the supervisory control proposition (Gable et al., 2015, 2018), anger should be positively and more strongly related to FAA than interest. Additionally, there is evidence that FAA predicts affective responding in the waking state (Coan and Allen, 2003) and is stable across waking and REM sleep (Benca et al., 1999; Schmidt et al., 2003), which indicates that FAA is not only a state- but also a trait-like marker of affective processing. Therefore, our second aim was to investigate whether waking FAA in the evening predicts affective experiences, specifically interest and anger, in REM sleep dreams. As such, this study helps clarify not only the neural correlates of affect during REM sleep dreaming, but also the extent to which these neural substrates are continuous with presleep wakefulness.

\section{Materials and Methods}

Participants. Data were collected within the framework of a larger study (Sikka et al., 2014). Altogether, 159 persons volunteered for the study (via advertisements posted in student mailing lists) and were prescreened using a background questionnaire. Participants fulfilling the following criteria were selected: healthy, not using medication, right-handed, native Finnish speakers, good sleep quality (score $\leq 5$ on the Pittsburgh Sleep Quality Index; Buysse et al., 1989). Because the present study focused on the relationship between (REM sleep and evening waking) FAA and affect experienced during REM sleep dreams (i.e., state affect), participants were not screened for trait affect. The selected 53 participants were asked to keep a $7 \mathrm{~d}$ home dream diary to measure spontaneous dream recall frequency. Twenty-two individuals returned the diary. Based on the number and word count of dream reports in the diary, participants were divided into two dream recall groups: high and low recallers. After having excluded 1 participant, high- and low-recall groups did not differ in their waking memory abilities, as measured with a range of memory tests (for details, see Sikka et al., 2014). However, all participants turned out to be good recallers in the laboratory setting (for results, see Sikka et al., 2014), and the two groups did not differ in any of the affect ratings or other variables included in this study ( $p$ values $>$ $0.05)$. Of the 21 participants who were invited, 19 accepted to sleep in the sleep laboratory. Due to technical problems, the data of 2 participants 


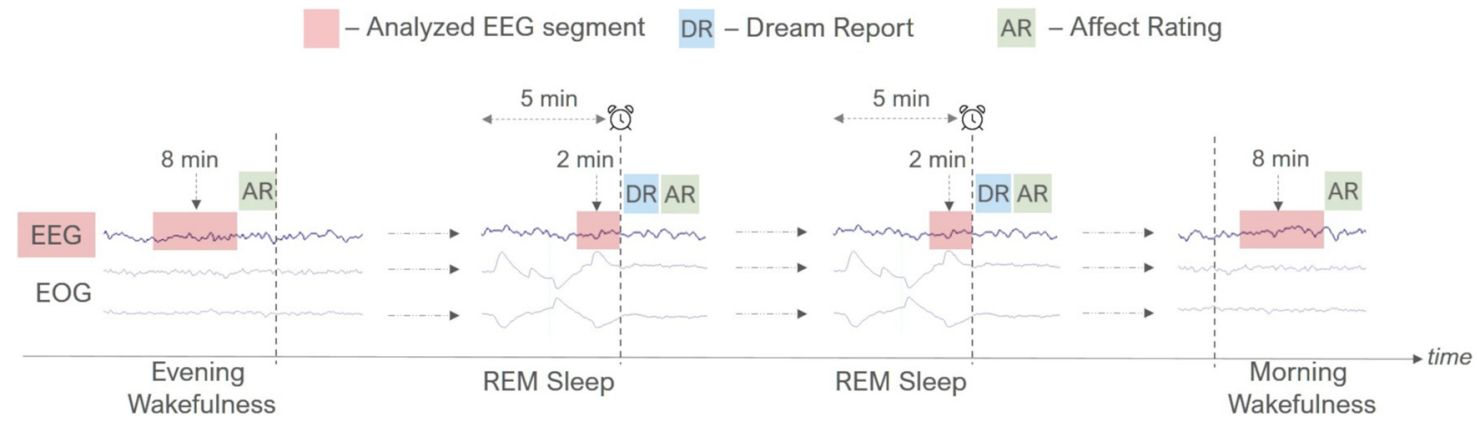

Figure 1. Description of the experimental procedure. In the evening, participants' waking state resting EEG was recorded for 8 min, after which they were asked to rate their current waking affect (AR). Participants were then allowed to fall asleep. Every time REM sleep had lasted continuously for $5 \mathrm{~min}$, and was in a phasic stage, a tone signal was used to awaken the participants. Upon awakening, participants provided an oral dream report (DR) and rated their dream affect (AR). They were then allowed to continue their sleep. This procedure was repeated throughout the night until the final morning awakening. Upon final awakening in the morning, participants' waking state resting EEG was again recorded for 8 min, followed by participants' ratings of their current waking affect (AR). Two minutes of preawakening REM sleep EEG that preceded each dream report, as well as the 8 min EEG during the evening and morning wakefulness, were analyzed. FAA (F4 -F3) during REM sleep and during evening wakefulness was used to predict dream affect ratings (AR).

were excluded. As a result, the final sample consisted of 17 participants $(7$ men, age $25.76 \pm 4.93$ years).

Experimental design and procedure. Participants spent 2 nights (separated by a week) in the sleep laboratory. A summary of the experimental procedure is displayed in Figure 1. In the evening, participants arrived in the sleep laboratory $2 \mathrm{~h}$ before their usual bedtime. After instructing the participants about the procedure of the study, EEG electrodes were attached to the scalp, and the waking state resting (evening baseline) EEG was recorded for eight 1 min periods, 4 min with eyes open (EO) and 4 min with eyes closed (EC) (order counterbalanced within and between participants). Baseline recordings were obtained in between 10:30 P.M. and 12:00 midnight while participants were lying in bed in the laboratory bedroom. Instructions to participants were given via intercom from an adjacent room. After the 8 min waking baseline EEG recording, participants rated their current waking affective state using the Finnish version of the modified Differential Emotions Scale (fmDES; see Measures).

Participants were then allowed to fall asleep. Sleep stages were scored visually (Rechtschaffen and Kales, 1968; Iber et al., 2007). Every time REM sleep had lasted continuously for $5 \mathrm{~min}$, and was in a phasic stage, a tone signal was used to awaken the participants. Upon awakening, participants provided an oral dream report according to the instructions they had received in the evening: they were first asked to report the last image they had in mind just before awakening, followed by a detailed report of the whole dream. Next, participants rated their affective experiences in the preceding dream by filling in the fmDES electronically using a mouse and a computer screen above the bed. In case participants reported no dream recall, fmDES was not filled in. Participants were then allowed to continue their sleep. This procedure was repeated throughout the night until the final morning awakening (scheduled between 5:30 A.M. to 8:30 A.M. in agreement with the participant). Upon final awakening, and after having reported and rated the last dream, participants were asked to lie in bed but stay awake. Similar to the evening, waking state resting (morning baseline) EEG was then recorded for $8 \mathrm{~min}$, followed by participants' ratings of their current waking state affect using the fmDES. Altogether, 126 awakenings (mean \pm SD, $7.41 \pm 3.02$ ) and $115(6.76 \pm 3.05)$ dream reports were obtained across the 2 nights. During the first night, 1 participant had only a single awakening that resulted in no dream recall. Thus, data regarding REM sleep and dream affect derived from 16 participants in the first night, and from 17 participants in the second night.

Signed informed consent was obtained from all participants. The study was performed in accordance with the Declaration of Helsinki and was approved by the Ethical Board of the University of Turku, Finland. Upon completion of the study, participants received 100 euros as compensation for their time.

Measures. Participants rated their waking and dream affect using the fmDES (Fredrickson, 2013). The scale consists of 20 items: 10 items for positive affect and 10 items for negative affect (with each item containing three adjectives). Each item was rated on a scale from 0 ("I did not experience any of these feelings at all") to 4 ("I experienced one or more of these feelings extremely much"). The current study focused on interest (as measured with the item Interested/Alert/Curious) and anger (as measured with the item Angry/Irritated/Annoyed). The other 18 items (see Table 2) were included in the exploratory analyses. For each participant, the mean dream affect rating was calculated across the different REM sleep episodes (and related dreams) for each laboratory night and for each item separately.

For exploratory analyses of externally rated dream anger, all oral dream reports $(N=115)$ were transcribed and content analyzed by two judges using the fmDES. The judges identified every time an affect was expressed in the dream report or could be unambiguously inferred from the behavior of the dream self. Then the judges categorized each occurrence of affect into 1 of the $20 \mathrm{fmDES}$ categories. The interrater reliability for the categorization of affect was strong (Cohen's $\kappa=0.84$ ) (for more details, see Sikka et al., 2014).

EEG recording, preprocessing, and analysis. A total of 24 single $\mathrm{Ag} / \mathrm{AgCI}$ electrodes (Fp1/2, AF3/4, AF7/8, F7/8, F3/4, Fz, T7/8, C3/4, Cz, P7/8, $\mathrm{P} 3 / 4, \mathrm{Pz}, \mathrm{O} 1 / 2, \mathrm{Oz}$ ) were placed on the participants' scalp according to the standard 10-10 system. Four electrodes were used to record EOG (two electrodes placed at the left and right outer canthi; one electrode superior and another inferior to the right eye), and two electrodes were used to record EMG (placed on the chin). All electrodes, except the bipolar EOG and EMG electrodes, were referenced to the right mastoid. The ground electrode was placed on the forehead. The EEG signal was amplified (SynAmps model 5083), notch-filtered at $50 \mathrm{~Hz}$, digitized at $500 \mathrm{~Hz}$, and recorded with Neuroscan equipment and software. All impedances were kept $<5 \mathrm{k} \Omega$.

Two minutes of preawakening EEG that preceded a dream report were extracted from each REM episode. These REM sleep EEG segments as well as the evening and morning 8 min baseline EEG segments from the 2 laboratory nights were processed offline using MATLAB (The MathWorks) and the EEGLAB toolbox version 14.1.1 (Delorme and Makeig, 2004).

EEG data were preprocessed according to the guidelines of Smith et al. (2017) using custom-made scripts. First, the extracted EEG segments were visually inspected and all nonbiological signals (except ocular and myogenic artifacts) rejected. EEG data were bandpass filtered using 0.5 $\mathrm{Hz}$ and $45 \mathrm{~Hz}$ cutoff values (FIR filters using the pop_eegfiltnew function; Delorme and Makeig, 2004). EOG and EMG as well as bad channels (marked by visual inspection) were removed. From the evening and morning baseline EEG, $4 \times 1$ min EO and $4 \times 1$ min EC conditions were extracted and processed separately. The continuous EEG signal was then segmented into $2 \mathrm{~s}$ epochs with 50\% overlap (Hamming window) and noisy epochs removed. Data were rereferenced to an average reference and baseline-corrected. Independent component analysis was computed and a semiautomatic procedure was used to remove artifactual components: the Multiple Artifact Rejection Algorithm (Winkler et al., 2011) extension of EEGLAB was used to highlight artifactual components and 
Table 1. Descriptive statistics for FAA and affect ratings ${ }^{a}$

\begin{tabular}{|c|c|c|c|c|c|c|c|c|c|}
\hline & Evening & & & REM sle & & & Morning & ness & \\
\hline & Mean & SE & $95 \% \mathrm{Cl}$ & Mean & SE & $95 \% \mathrm{Cl}$ & Mean & SE & $95 \% \mathrm{Cl}$ \\
\hline FAA & -0.08 & 0.10 & {$[-0.30,0.14]$} & -0.13 & 0.07 & {$[-0.28,0.01]$} & -0.29 & 0.11 & {$[-0.53,-0.04]$} \\
\hline Anger & 0.26 & 0.09 & {$[0.15,0.41]$} & 0.64 & 0.10 & {$[0.46,0.83]$} & 0.15 & 0.06 & {$[0.06,0.24]$} \\
\hline Interest & 1.26 & 0.19 & {$[0.91,1.62]$} & 1.61 & 0.15 & {$[1.36,1.87]$} & 0.88 & 0.17 & {$[0.59,1.18]$} \\
\hline
\end{tabular}

${ }^{a}$ Bias-corrected accelerated bootstrap Cls based on 5000 bootstrap samples. FAA scores $\left(8-13 \mathrm{~Hz}\right.$ at F4-F3) are in $\mu \mathrm{V} / \mathrm{cm}^{2}$ units. Anger and interest scores reflect the mean intensity on a $0-4$ rating scale.

those containing ocular and myogenic artifacts (confirmed by visual inspection) were removed. Artifactual components were then subtracted from the data. Previously removed channels were interpolated. Artifactfree epochs were transformed into current source density estimates $(\mu \mathrm{V} /$ $\mathrm{cm}^{2}$ units; $10 \mathrm{~cm}$ head radius; $\mathrm{m}=4 ; \lambda=10^{-5}, 50$ iterations) using a spherical spline surface Laplacian transformation (Perrin et al., 1989) in the current source density toolbox (Kayser and Tenke, 2006a,b). Current source density is recommended because it is independent of the reference used, reduces volume conduction from distal sites, enhances spatial resolution of the EEG signal, and is also appropriate with low-density EEG montages (Kayser and Tenke, 2015; Smith et al., 2017). Then, a fast Fourier transform (using the MATLAB $f f t$ function) was applied to each artifact-free epoch to obtain the mean spectral power in the alpha frequency band $(8-13 \mathrm{~Hz})$ for each electrode. An average of 114.45 artifact-free REM sleep epochs and $216.74(\mathrm{EC}=216.00 ; \mathrm{EO}=217.47)$ artifact-free resting wakefulness epochs were analyzed for each participant.

Statistical analyses. Statistical analyses were conducted with R (version 3.4.1, R Development Core Team 2017) and SPSS (version 20, IBM). Alpha power spectra were averaged for each participant and for each condition (evening baseline EO, evening baseline EC, morning baseline EO, morning baseline EC, REM sleep) for the 2 laboratory nights separately. Alpha power at each electrode was natural log-transformed to normalize the data. FAA score was then calculated by subtracting logtransformed alpha power of the left hemisphere electrode from that of the homologous right hemisphere electrode (i.e., $\ln [\mathrm{F} 4]-\ln [\mathrm{F} 3]$ ). Positive values reflect relatively more alpha power in the right hemisphere (i.e., lower right-sided or greater left-sided frontal activity). The main analyses focused on the canonical pair of F4-F3 electrodes. However, to control for the spatial specificity of the observed effects, FAA was also calculated for all the other homologous pairs (i.e., $\ln [\mathrm{Fp} 2]-\ln [\mathrm{Fp} 1], \ln [\mathrm{AF} 8]-$ $\ln [\mathrm{AF} 7], \ln [\mathrm{AF} 4]-\ln [\mathrm{AF} 3], \ln [\mathrm{F} 8]-\ln [\mathrm{F} 7], \ln [\mathrm{T} 8]-\ln [\mathrm{T} 7], \ln [\mathrm{C} 4]-\ln [\mathrm{C} 3]$, $\left.\ln [\mathrm{P} 8]-\ln [\mathrm{P} 7], \ln [\mathrm{P} 4]-\ln [\mathrm{P} 3], \ln \left[\mathrm{O}_{2}\right]-\ln [\mathrm{O} 1]\right)$.

Differences in the obtained FAA scores between the resting wakefulness EO and EC conditions and between the 2 laboratory nights were analyzed using paired-samples $t$ test (in case of normal distribution) or Wilcoxon signed-rank test (in case of non-normal distribution). The Shapiro-Wilk test (Shapiro and Wilk, 1965) was used to test the normality assumption. Because the FAA scores of the baseline EO and EC conditions did not differ from each other $(p$ values $>0.05$ ), these were aggregated to form one evening (EOEC) and one morning (EOEC) baseline score for each laboratory night. There were no significant differences between the 2 nights in the evening baseline (paired-samples $t_{(16)}=$ $-0.264, p=0.796, N=17$ ), morning baseline (paired-samples $t_{(16)}=$ $0.826, p=0.421, N=17$ ) and REM sleep FAA scores (paired-samples $\left.t_{(15)}=0.870, p=0.398, N=16\right)$. There were also no differences between the 2 nights in the ratings of dream anger (paired-samples $t_{(15)}=1.404$, $p=0.181, N=16)$ and dream interest (paired-samples $t_{(15)}=0.193, p=$ $0.850, N=16$ ), or in the ratings of evening anger (Wilcoxon $Z=-0.632$, exact $p=0.766, N=17$ ), evening interest (Wilcoxon $Z=-0.676$, exact $p=0.640, N=17$ ), morning anger (Wilcoxon $Z=-1.342, p=0.375$, $N=17$ ), and morning interest (Wilcoxon $Z=-0.061, p=1.000, N=$ 17). Therefore, the FAA and dream affect scores from the 2 nights were pooled together. Also, there were no differences between males and females in anger or interest ratings or in FAA scores in any of the conditions (using independent-samples $t$ tests or Mann-Whitney $U$ tests; $p$ values $>$ $0.05)$. As such, the analyses and results reported below are based on the pooled (average of 2 nights) scores, including both genders.

To ease reading, the specific methods used for statistical analyses are reported below in conjunction with respective results. Parametric tests
(ANOVA, paired-samples $t$ test, simple and multiple linear regression, correlation and partial correlation) were used when the scores followed a normal distribution; whereas for non-normal distribution of scores, nonparametric tests (Wilcoxon signed-rank test, Spearman rank correlation, and nonparametric partial correlation) were used. In all the analyses, we considered $p$ values $<0.05$ as statistically significant. For regression analyses we report unstandardized coefficients (B), confidence intervals for unstandardized coefficients, and standard errors for unstandardized coefficients. Where appropriate, we report effect sizes in the form of $r$ values ( $r=\mathrm{Z} / \sqrt{ } \mathrm{N}$; Wilcoxon signed-rank test), Cohen's $d$ (paired-samples $t$ test), or partial $\eta$ squared ( $\eta p^{2}$; ANOVA). All statistical tests were two-tailed and for nonparametric tests exact tests were conducted. The number of participants in all the analyses reported below was 17.

\section{Results}

Anger and interest across wakefulness and REM sleep dreams Anger was experienced in $41 \%$ of dreams, interest in $88 \%$ of dreams. Participants experienced more anger in dreams than during the evening (Wilcoxon $Z=-2.354$, exact $p=0.016, r=$ 0.40 ) or morning (Wilcoxon $Z=-3.125$, exact $p=0.001, r=$ 0.54 ) wakefulness, whereas the evening and morning anger ratings did not differ (Wilcoxon $Z=-1.134$, exact $p=0.500, r=$ 0.19 ) (Table 1; Fig. $2 A$ ). Anger ratings across the three conditions were not correlated ( $p$ values $>0.05$ ).

Participants experienced more interest during dreaming (Wilcoxon $Z=-2.897$, exact $p=0.002, r=0.49$ ) and during the evening wakefulness (Wilcoxon $Z=-2.360$, exact $p=0.022, r=$ 0.41 ), compared with morning wakefulness. There were no differences in the ratings of interest between the evening wakefulness and dreams (paired-samples $t_{(16)}=-1.534, p=0.145, d=$ $0.38)$. Ratings of interest were correlated between the evening and morning wakefulness $\left(r_{s}=0.732, p=0.001\right)$ but not between dream and wakefulness conditions ( $p$ values $>0.05$ ).

Thus, participants experienced more intense affective states during dreaming than during presleep or postsleep resting wakefulness.

\section{FAA across wakefulness and REM sleep}

A one-way repeated-measures ANOVA (within-subject factor: condition) showed that the mean FAA score differed between the three conditions $\left(F_{(2,32)}=3.371, p=0.047, \eta p^{2}=0.174\right)$. Post hoc least significance difference tests revealed that, whereas the morning FAA score was significantly more negative than the evening FAA score $(p=0.025)$, REM sleep FAA score did not differ from the evening $(p=0.507)$ or morning $(p=0.102)$ FAA scores (Table 1). FAA scores were positively correlated across all the conditions, between evening and REM sleep $(r=0.701, p=$ $0.002)$, REM sleep and morning $(r=0.637, p=0.006)$, and evening and morning $(r=0.720, p=0.001)$, indicating a strong association of FAA scores across the behavioral states.

\section{Relationship between dream anger, dream interest, and REM sleep FAA}

To investigate the relationship between REM sleep FAA and dream affect, separate linear regression analyses were performed 
(A)

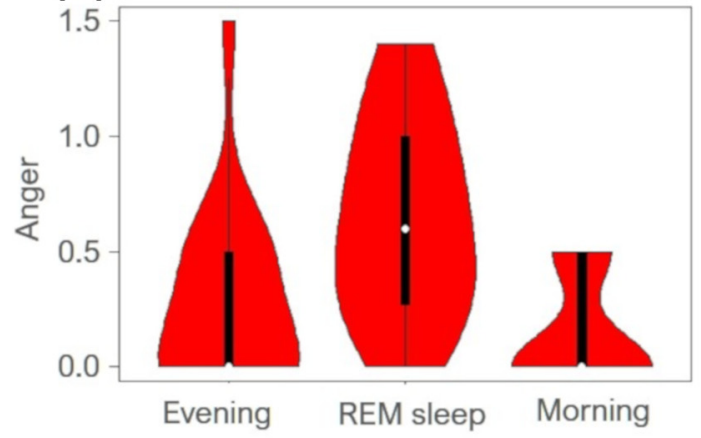

(C)

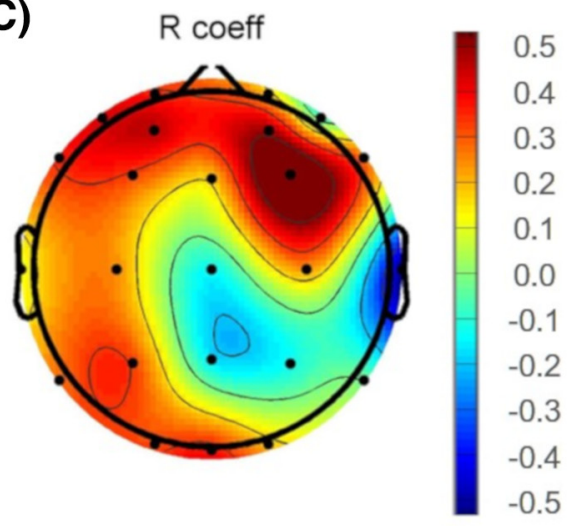

(E)

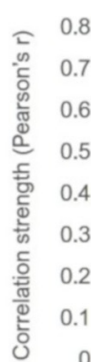

(B)

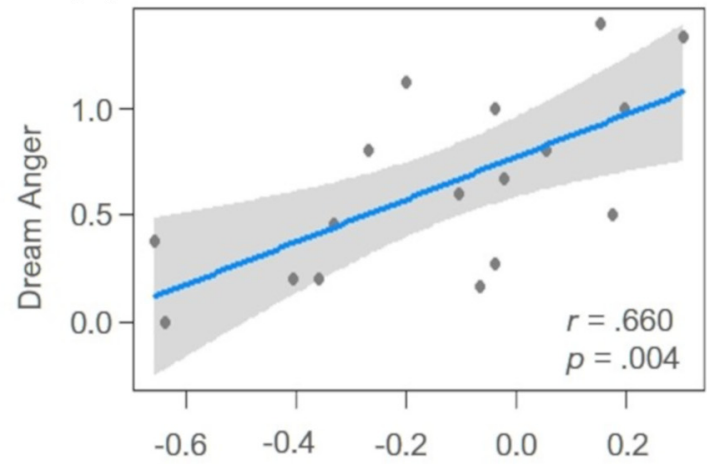

REM Sleep Frontal Alpha Asymmetry $\ln (F 4)-\ln (F 3)$

(D)

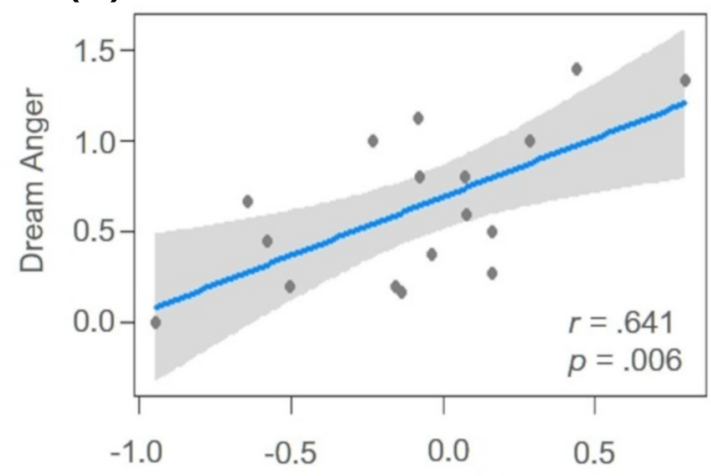

Evening Waking Frontal Alpha Asymmetry $\ln (F 4)-\ln (F 3)$

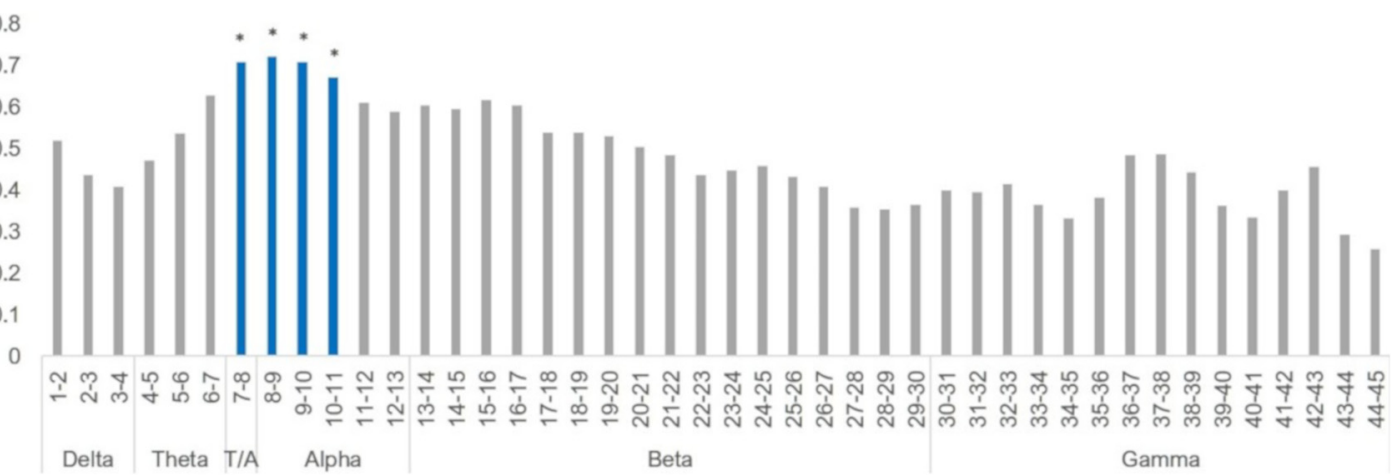

Figure 2. Dream anger and its relationship to FAA.A, Violin plot displaying ratings of anger in the evening resting wakefulness, REM sleep dreams, and morning resting wakefulness. Middle white dot represents the median value. Middle thick black bar represents the interquartile range. Thin gray line indicates $95 \%$ Cl. B, Relationship between dream anger and REM sleep FAA. Gray area represents $95 \% \mathrm{CI}$. C, Partial correlation coefficients between dream anger and log-transformed alpha power over individual electrode sites while controlling for the average whole-head alpha power. $\boldsymbol{D}$, Relationship between dream anger and FAA during evening resting wakefulness. Gray area represents $95 \% \mathrm{Cl}$. E, Partial correlation coefficients between dream anger and log-transformed alpha power over the $\mathrm{F} 4$ electrode in $1 \mathrm{~Hz}$ bins. Blue-colored bins marked with an asterisk represent statistically significant correlations after the Benjamini-Hochberg False Discovery Rate correction (adjusted $p$ value $=0.0045)$. T/A, theta/alpha.

in which ratings of dream anger and dream interest were regressed on the FAA score. Results showed that REM sleep FAA was a significant predictor of anger in dreams $\left(R^{2}=0.435, \mathrm{~B}=\right.$ $0.993,95 \%$ CI $[0.370,1.616], \mathrm{SE}=0.292, t=3.398, p=0.004)$ (Fig. 2B).

To assess the spatial specificity of the observed effect, a multiple linear regression analysis was conducted in which the wholehead alpha power (average of all electrodes) was entered together with the natural log-transformed alpha power of F3 and F4 electrodes (as suggested in Allen et al., 2004). This controls for the overall alpha power in predicting anger in dreams. Results showed that the overall model was significant $\left(R^{2}=0.604\right.$, $\left.F_{(3,13)}=6.606, p=0.006\right)$. However, only the right frontal region (F4) was a significant predictor of dream anger $(\mathrm{B}=$ $0.960,95 \%$ CI $[0.391,1.529], \mathrm{SE}=0.263, t=3.646, p=$ $0.003)$. The left frontal region (F3) ( $\mathrm{B}=-0.551,95 \% \mathrm{CI}$ $[-1.332,0.229], \mathrm{SE}=0.361, t=-1.526, p=0.151)$ and the average whole-head alpha power $(\mathrm{B}=-284.744,95 \% \mathrm{CI}$ $[-1739.107,1169.619], \mathrm{SE}=673.201, t=-0.423, p=0.679)$ were not significant. This indicates that the relationship be- 
tween REM sleep FAA and dream anger was driven by higher alpha power in the right frontal region.

To control for the cerebral specificity of the observed relationship, we repeated the analyses using the removed independent component analysis components reflecting ocular and myogenic artifacts. Results showed that there were no significant relationships $\left(R^{2}=0.002, \mathrm{~B}=0.048,95 \% \mathrm{CI}[-0.571,0.667], \mathrm{SE}=\right.$ $0.290, t=0.166, p=0.871$ ) between ratings of dream anger and FAA calculated using these artifactual components. This provides support to the cerebral (rather than ocular or myogenic) origin of the findings.

To explore whether the relationship between asymmetric cortical activity during REM sleep and dream anger was specific to the mid-frontal region, we performed exploratory correlation analyses for dream anger and all the different hemispheric asymmetry scores (e.g., $\ln [\mathrm{F} 8]-\ln [\mathrm{F} 7])$. None of the other hemispheric asymmetry scores was significantly related to dream anger ( $p$ values $>0.05$ ). Partial correlations between dream anger and logtransformed alpha power over individual electrode sites, while controlling for the average whole-head alpha power, showed that, in addition to the significant correlation between dream anger and alpha power over the right frontal electrode F4 $(r=0.691$; $p=0.003$ ), the only other significant correlation occurred between dream anger and alpha power over the right temporal electrode T8 ( $r=-0.528, p=0.035)$ (Fig. $2 C)$.

We conducted additional exploratory analyses to investigate whether the relationship between right-sided frontal activity and dream anger was specific to alpha band only. For this, we repeated the partial correlation analyses between dream anger and logtransformed alpha power over the $\mathrm{F} 4$ electrode site in $1 \mathrm{~Hz}$ frequency bins from 1 to $45 \mathrm{~Hz}$, while controlling for the average whole-head alpha power. Results showed that, after controlling for multiple comparisons using the Benjamini-Hochberg false discovery rate $(q<0.05$; Benjamini and Hochberg, 1995), only the frequency bins $7-8 \mathrm{~Hz}(r=0.708, p=0.002), 8-9 \mathrm{~Hz}(r=$ $0.721, p=0.002), 9-10 \mathrm{~Hz}(r=0.712, p=0.002)$, and $10-11 \mathrm{~Hz}$ $(r=0.672, p=0.004)$ over F4 were significantly correlated with dream anger (Fig. 2E). No significant correlations occurred between similar analyses involving F3 ( $p$ values $>0.05$ ). Thus, the relationship between dream anger and right-sided cortical activity was specific to lower alpha band $(7-11 \mathrm{~Hz})$ only.

Furthermore, we explored whether the results only apply to self-ratings of dream anger (i.e., participants' own ratings of anger experienced in the preceding dream) or also to the so-called external ratings of dream anger (i.e., anger identified in the participants' dream reports by external judges). Although Spearman correlation analyses showed no significant relationships between FAA and externally rated dream anger ( $p$ values $>0.05$ ), nonparametric partial correlation analyses between dream anger and log-transformed alpha power over individual electrode sites (while controlling for the average whole-head alpha power) revealed that anger expressed in dream reports was significantly related to alpha power over F4 ( $r=0.535, p=0.033)$, but not F3 $(r=0.385, p=0.141)$. This provides evidence that the relationship between right-sided frontal alpha and dream anger was not dependent on the particular method used for rating dream affect.

REM sleep FAA did not predict ratings of dream interest $(r=$ $0.444, R^{2}=0.198, \mathrm{~B}=0.995, \mathrm{SE}=0.518,95 \%$ CI $[-0.109$, 2.099], $t=1.922, p=0.074$ ).

We also performed exploratory analyses to investigate whether any of the other dream affect ratings was related to REM sleep FAA. Spearman correlation analysis showed that the only other item related to REM sleep FAA was Hate/Distrust/Suspi-
Table 2. Spearman correlation coefficients between REM sleep FAA and dream affect $^{a}$

\begin{tabular}{lc}
\hline Discrete dream affect & REM sleep FAA \\
\hline PA1 Amused/Fun-loving/Giggly & -0.006 \\
PA2 Awe/Wonder/Amazement & -0.235 \\
PA3 Grateful/Appreciative/Thankful & -0.165 \\
PA4 Hopeful/Optimistic/Encouraged & 0.020 \\
PA5 Inspired/Uplifted/Elevated & 0.131 \\
PA6 Interested/Alert/Curious & 0.405 \\
PA7 Joyful/Glad/Happy & 0.218 \\
PA8 Love/Closeness/Trust & -0.031 \\
PA9 Proud/Confident/Self-assured & 0.050 \\
PA10 Serene/Content/Peaceful & 0.106 \\
NA1 Angry//rritated/Annoyed & $0.638^{* *}$ \\
NA2 Ashamed/Humiliated/Disgraced & 0.080 \\
NA3 Contemptuous/Scornful/Disdainful & 0.114 \\
NA4 Disgust/Distaste/Revulsion & 0.431 \\
NA5 Embarrassed/Self-conscious/Blushing & 0.276 \\
NA6 Guilty/Repentant/Blameworthy & 0.255 \\
NA7 Hate/Distrust/Suspicion & $0.572^{*}$ \\
NA8 Sad/Downhearted/Unhappy & -0.020 \\
NA9 Scared/Fearful/Afraid & 0.116 \\
NA10 Stressed/Nervous/Overwhelmed & -0.198 \\
\hline
\end{tabular}

${ }^{a} \mathrm{NA}$, Negative affect item; PA, positive affect item.

${ }^{*} p=0.016 ;{ }^{* *} p=0.006$.

cion $\left(r_{s}=0.572, p=0.016\right.$; Table 2$)$. As this item was strongly related to the item Angry/Annoyed/Frustrated $\left(r_{s}=0.648, p=\right.$ 0.005 ), it provides further support for the specific relationship between FAA and anger in dreams. Originally, this item was not included in the analyses because of the adjectives distrust/suspicion, which do not make it a pure anger and high-approach negative affect item.

\section{Relationship between dream anger, dream interest, and waking state FAA}

To investigate the relationship between evening FAA and dream affect, ratings of dream anger and interest were regressed on the evening FAA score. Results showed that evening FAA score was a significant predictor of dream anger $\left(R^{2}=0.411, \mathrm{~B}=0.644,95 \%\right.$ $\mathrm{CI}[0.220,1.068], \mathrm{SE}=0.199, t=3.236, p=0.006)($ Fig. $2 D)$ but not of dream interest $\left(R^{2}=0.016, \mathrm{~B}=0.189,95 \%\right.$ CI $[-0.626$, $1.004], \mathrm{SE}=0.382, t=0.495, p=0.628)$.

Because evening FAA and REM sleep FAA both predicted anger in dreams, while also being strongly correlated with each other, we performed two sets of partial correlation analyses to further explore the relationship between FAA and dream anger. When controlling for evening FAA, the relationship between REM sleep FAA and dream anger was no longer significant $(r=$ $0.384, p=0.142$ ). The same was true for the relationship between evening FAA and dream anger $(r=0.334, p=0.206)$, when controlling for REM FAA. This shows that the relationship between dream anger and FAA in one condition was fully accounted for by FAA in the other condition; that is, evening and REM sleep FAA overlapped.

Ratings of dream anger $\left(R^{2}=0.208, \mathrm{~B}=0.508,95 \% \mathrm{CI}\right.$ $[-0.037,1.053], \mathrm{SE}=0.256, t=1.986, p=0.066)$ and dream interest $\left(R^{2}=0.047, \mathrm{~B}=0.162,95 \% \mathrm{CI}[-0.241,0.564], \mathrm{SE}=\right.$ $0.189, t=0.856, p=0.406)$ did not predict morning FAA.

\section{Relationship between waking FAA and waking affect}

Evening and morning waking baseline FAA scores were not correlated with waking affect ( $p$ values $>0.05$ ), probably due to the low level of affect experienced in these states. 


\section{Discussion}

We investigated whether affective dream experiences, specifically anger and interest, are related to FAA-hemispheric differences in alpha-band oscillations between the right and left frontal cortical regions. Results showed that FAA during REM sleep predicted ratings of dream anger: participants with greater relative alpha power in the right (F4), compared with the left (F3), frontal region experienced more anger in dreams. Because alpha power is taken to reflect reduced activity in underlying areas (Klimesch et al., 2007; Jensen and Mazaheri, 2010; Klimesch, 2012), and because the relationship was found to be specifically driven by the right hemisphere, the results suggest that anger is related to reduced activity in the right frontal region. This is further supported by the findings that the only other dream affect rating significantly correlated with REM sleep FAA was an item closely related to dream anger-Hate/Distrust/Suspicion. Additionally, we found that FAA recorded during evening resting wakefulness predicted anger in dreams. These findings are in line with several studies conducted on FAA and anger in the waking state (e.g., Harmon-Jones and Sigelman, 2001; Jensen-Campbell et al., 2007; for review, see Harmon-Jones and Gable, 2018).

Moreover, these results were specific to frontal regions only (F4-F3). Although in the present study the exact anatomical source for FAA remains unclear, previous studies conducted in the waking state have shown that FAA originates from the dorsolateral PFC (Ellis et al., 2017) and from the lateral and medial frontal gyri (Gable et al., 2015; Neal and Gable, 2016). This suggests that dream anger is most likely related to reduced activity in the right lateral PFC (IPFC). REM sleep is typically associated with the deactivation of IPFC (Maquet et al., 1996; Braun et al., 1998); and given the importance of $1 \mathrm{PFC}$ in functions related to cognitive control, this helps explain enhanced anger in dreams. However, deactivation of IPFC is not an all-or-none phenomenon, and there is also evidence for sustained (Vijayan et al., 2017) and even increased activation of IPFC in REM sleep, compared with wakefulness (Nofzinger et al., 1997; Wehrle et al., 2007). In line with these findings, our study demonstrates that there are individual differences in the asymmetric activity of IPFC during REM sleep, and this is differentially related to dream anger.

The finding that dream anger (a high-approach negative affect), but not dream interest (a high-approach positive affect), was related to FAA corroborates the theory that FAA reflects supervisory control (Gable et al., 2015, 2018), rather than affective valence or motivational direction. As such, individuals with reduced right PFC activity (as indicated by more alpha power) may be less able to regulate (i.e., inhibit) strong affective states, such as anger, not only during wakefulness but also during dreaming. Because the regulation of negative affect is more resource consuming than the regulation of positive affect (Ochsner et al., 2004), individuals with reduced right PFC activity may have problems specifically with regulating negative affective states. This is supported by studies showing that the downregulation (i.e., control) of negative affect, compared with positive affect, uniquely involves right-lateralized PFC regions (Kim and $\mathrm{Ha}-$ mann, 2007).

It is important to note that the right PFC is involved not only in the regulation of negative affect, but also in inhibitory control in general (Bari and Robbins, 2013; Aron et al., 2014; Depue et al., 2016). Numerous studies conducted in the waking state have demonstrated an association between greater FAA (i.e., relatively less right frontal activity) and states and traits related to disinhibition, such as aggression (Harmon-Jones and Allen, 1998;
Harmon-Jones and Sigelman, 2001), impulsivity (Gable et al., 2015; Neal and Gable, 2017), sensation seeking (Santesso et al., 2008), (positive and negative) urgency (Neal and Gable, 2016), response inhibition (Ellis et al., 2017), and risk-taking behavior (Gianotti et al., 2009). Moreover, studies using transcranial direct current stimulation and repetitive transcranial magnetic stimulation have shown that inhibition of the right, or stimulation of the left, IPFC leads to disinhibition (for review, see Kelley et al., 2017). Therefore, it may well be that enhanced anger in dreams reflects a more general aspect of disinhibition. At the same time, it may also be the case that other affective states in dreams (e.g., fear) are also related to FAA; but because of a very low incidence of these in the current dream sample, their statistical assessment was not robust.

The finding that FAA was strongly correlated across wakefulness and REM sleep is in line with previous studies that have demonstrated the stability of FAA in wakefulness (Coan and Allen, 2003), and across wakefulness and different sleep stages (Benca et al., 1999; Schmidt et al., 2003). This result, together with the finding that presleep waking FAA predicted dream anger, indicates that FAA has trait-like properties. This is further supported by the overlap between the evening FAA and REM sleep FAA in their association with dream anger. Indeed, based on research in the waking state, it has been suggested that, whereas $\sim 40 \%$ of the variation in FAA reflects state-like influences, $\sim 60 \%$ reflects trait-like influences (Hagemann et al., 2002). This may also be one possible explanation as to why we did not observe any relationships between waking resting FAA and waking affect ratings. It has been argued that trait-like individual differences in FAA may be most apparent in a situation that elicits affective states (Coan et al., 2006). Because in the current study participants' ratings of waking affect were low, individual differences in FAA may have been reflected most clearly during states high in affectivity (i.e., during dreaming). Therefore, future studies should measure waking FAA during states of high affectivity (e.g., after experimental induction of affective states) and then compare the FAA-affect relationship obtained during wakefulness to that obtained during dreaming. The trait-like nature of FAA suggests that it may reflect a trait-like predisposition to experience certain affective states, such as anger, not only during wakefulness but also during dreaming. To directly test this proposition, future research should include repeated measurements of FAA and affective experiences across different wakefulness periods and sleep stages (including non-REM sleep), and explore how trait affect (e.g., trait anger) or trait affect regulation measures relate to FAA and affect experienced across those states. This will help clarify whether FAA can be considered as a marker of stateor trait-like affective processing across different states of consciousness (see also Scarpelli et al., 2015a,b).

Overall, our findings show that anger in REM sleep dreams shares the same neural correlates as those observed in wakefulness and that these neural substrates are continuous with presleep wakefulness. Together with other neural studies of dream content (Dresler et al., 2011; Horikawa et al., 2013; Perogamvros et al., 2017; Siclari et al., 2017), the converging evidence suggests highly overlapping neurobiological mechanisms of conscious subjective experience in the two major global states of consciousness-waking and dreaming - regardless of the lack of responsiveness in the latter (see also De Gennaro et al., 2012; Fox et al., 2013; Wamsley, 2013; Domhoff and Fox, 2015). As such, these findings provide support for theories according to which dreaming is a realistic simulation of waking life (e.g., Foulkes, 1985; Nielsen, 2010; Hobson and Friston, 2012; Windt, 2015; Revonsuo et al., 2016; 
Domhoff, 2018). However, based on the present study, it is not possible to say whether the particular neural activation accompanying dream anger supports a certain function, such as to experience threatening situations or negative affective states to better deal with them in waking life (e.g., Revonsuo, 2000; Nielsen and Levin, 2007; Cartwright, 2010; Perogamvros and Schwartz, 2012), to consolidate specific types of memories (Wamsley and Stickgold, 2011), or whether it simply reflects the individual's waking life experiences (Domhoff, 1996, 2017; Schredl, 2003).

Future studies could probe the causal nature of the observed interaction by using various brain stimulation techniques, such as transcranial direct current stimulation or repetitive transcranial magnetic stimulation. It would be interesting to explore whether the activation (vs inhibition) of the right lPFC during REM sleep and presleep wakefulness would lead to reduced (vs increased) anger in dreams. This may have clinical implications because anger is frequently experienced during nightmares and frequent nightmares are associated with several different mental (e.g., anxiety, depression) and sleep (e.g., insomnia) disorders (Levin and Nielsen, 2007; Nielsen and Levin, 2007; Skancke et al., 2014). Moreover, the treatment of nightmares has been shown to improve symptoms of post-traumatic stress disorder (Spoormaker and Montgomery, 2008). Therefore, finding ways to modulate negative affective experiences may lead to novel interventions.

Regarding possible limitations of the present study, it could be argued that, because dream affect ratings were performed in the waking state (i.e., upon awakening from the dream), these do not reflect affective experiences in dreams but during the time the reports were given. Perhaps individuals with higher FAA scores (i.e., more alpha power in the right frontal area) did not actually experience more anger in dreams but were simply predisposed to rate their dreams in a more negative manner. If so, such a bias should also apply to ratings of waking affect. However, given that the ratings of affect (especially anger) were much lower during evening and morning wakefulness and the fact that the ratings of dream affect did not correlate with those given in the waking state, this explanation seems unlikely. Moreover, additional analyses demonstrated that right-sided frontal alpha power was related not only to self-ratings of dream anger (i.e., how participants themselves rated the anger they experienced in the preceding dream) but also to external ratings of dream anger (i.e., anger expressed by participants in corresponding dream reports as identified by external raters). This is especially important in light of recent studies showing that the method used to rate dream affect (i.e., self-ratings vs external ratings) can yield different results regarding dream affectivity (Schredl and Doll, 1998; Sikka et al., 2014, 2017). Thus, our results are not dependent on the particular dream affect rating method which provides further credence for our findings.

Another limitation is that, while dream affect ratings were preceded by a narrative report of dream experiences, no such tasks preceded waking affect ratings. It is possible that the act of reflecting on or reporting experiences before rating them may have influenced the ratings. Thus, future studies should also include narrative (i.e., mind-wandering) reports in the waking state.

Additionally, because the current study was performed in the laboratory environment, the findings may have been influenced by the experimental setting and procedure. For example, it has been shown that home dream reports contain more negative affect, especially fear, than laboratory dream reports (Foulkes, 1979; Sikka et al., 2018). As such, it would be important to repli- cate the study in a more naturalistic home environment. Furthermore, given the selection criteria and the number of participants, it is important to replicate this study in larger and more diverse samples, and to control for possible individual differences in the ability to identify and describe affective states (e.g., alexithymia).

In conclusion, this is the first study on the relationship between discrete affective states in dreams and FAA. Results show that FAA during REM sleep as well as during evening wakefulness predicts anger in dreams. These findings imply that affective experiences may rely on the same neural processes across wakefulness and REM sleep despite the different neurophysiological bases of these states. Specifically, the results suggest that FAA may serve as a neural correlate of affect regulation (i.e., inhibitory control of affective processing) not only in the waking but also in the dreaming state.

\section{References}

Allen JJ, Coan JA, Nazarian M (2004) Issues and assumptions on the road from raw signals to metrics of frontal EEG asymmetry in emotion. Biol Psychol 67:183-218.

Aron AR, Robbins TW, Poldrack RA (2014) Inhibition and the right inferior frontal cortex: one decade on. Trends Cogn Sci 18:177-185.

Bari A, Robbins TW (2013) Inhibition and impulsivity: behavioral and neural basis of response control. Prog Neurobiol 108:44-79.

Barrett LF (2004) Feelings or words? Understanding the content in self-report ratings of experienced emotion. J Pers Soc Psychol 87:266-281.

Benca RM, Obermeyer WH, Larson CL, Yun B, Dolski I, Kleist KD, Weber SM, Davidson RJ (1999) EEG alpha power and alpha power asymmetry in sleep and wakefulness. Psychophysiology 36:430-436.

Benjamini Y, Hochberg Y (1995) Controlling for the false discovery rate: a practical and powerful approach to multiple testing. J R Stat Soc B 57: 289-300.

Blake Y, Terburg D, Balchin R, van Honk J, Solms M (2019) The role of the basolateral amygdala in dreaming. Cortex 113:169-183.

Braun AR, Balkin TJ, Wesensten NJ, Gwadry F, Carson RE, Varga M, Baldwin P, Belenky G, Herscovitch P (1998) Dissociated pattern of activity in visual cortices and their projections during human rapid eye movement sleep. Science 279:91-95.

Buysse DJ, Reynolds CF 3rd, Monk TH, Berman SR, Kupfer DJ (1989) The Pittsburgh Sleep Quality Index: a new instrument for psychiatric practice and research. Psychiatry Res 28:193-213.

Cartwright R (2010) The twenty-four hour mind: the role of sleep and dreaming in our emotional lives. New York: Oxford UP.

Cipolli C, Ferrara M, De Gennaro L, Plazzi G (2017) Beyond the neuropsychology of dreaming: insights into the neural basis of dreaming with new techniques of sleep recording and analysis. Sleep Med Rev 35:8-20.

Coan JA, Allen JJ, McKnight PE (2006) A capability model of individual differences in frontal EEG asymmetry. Biol Psychol 72:198-207.

Coan JA, Allen JJ (2003) The state and trait nature of frontal EEG. In: The asymmetrical brain (Hughdahl K, Davidson RJ, eds), pp 565-615. Cambridge, MA: Massachusetts Institute of Technology.

Daoust AM, Lusignan FA, Braun CM, Mottron L, Godbout R (2008) EEG correlates of emotions in dream narratives from typical young adults and individuals with autistic spectrum disorders. Psychophysiology 45:299_ 308.

Davidson RJ (1998) Affective style and affective disorders: perspectives from affective neuroscience. Cogn Emot 12:307-330.

Davidson RJ, Schwartz GE, Saron C, Bennett J, Goleman DJ (1979) Frontal versus parietal EEG asymmetry during positive and negative affect. Psychophysiology 16:202-203.

De Gennaro L, Cipolli C, Cherubini A, Assogna F, Cacciari C, Marzano C, Curcio G, Ferrara M, Caltagirone C, Spalletta G (2011) Amygdala and hippocampus volumetry and diffusivity in relation to dreaming. Hum Brain Mapp 32:1458-1470.

De Gennaro L, Marzano C, Cipolli C, Ferrara M (2012) How we remember the stuff that dreams are made of: neurobiological approaches to the brain mechanisms of dream recall. Behav Brain Res 226:592-596.

Delorme A, Makeig S (2004) EEGLAB: an open source toolbox for analysis of single-trial EEG dynamics including independent component analysis. J Neurosci Methods 134:9-21. 
Depue BE, Orr JM, Smolker HR, Naaz F, Banich MT (2016) The organization of right prefrontal networks reveals common mechanisms of inhibitory regulation across cognitive, emotional, and motor processes. Cereb Cortex 26:1634-1646.

Desseilles M, Dang-Vu TT, Sterpenich V, Schwartz S (2011) Cognitive and emotional processes during dreaming: a neuroimaging view. Conscious Cogn 20:998-1008.

Domhoff GW (2018) The emergence of dreaming: mind-wandering, embodied simulation, and the default network. New York: Oxford UP.

Domhoff WG (1996) Finding meaning in dreams: a quantitative approach. New York: Plenum.

Domhoff WG (2017) The invasion of the concept snatchers: the origins, distortions, and future of the continuity hypothesis. Dreaming 27:14-39.

Domhoff GW, Fox KC (2015) Dreaming and the default network: a review, synthesis, and counterintuitive research proposal. Conscious Cogn 33:342-353.

Dresler M, Koch SP, Wehrle R, Spoormaker VI, Holsboer F, Steiger A, Sämann PG, Obrig H, Czisch M (2011) Dreamed movement elicits activation in the sensorimotor cortex. Curr Biol 21:1833-1837.

Ellis AJ, Kinzel C, Salgari GC, Loo SK (2017) Frontal alpha asymmetry predicts inhibitory processing in youth with attention deficit/hyperactivity disorder. Neuropsychologia 102:45-51.

Foulkes D (1979) Home and laboratory dreams: four empirical studies and a conceptual reevaluation. Sleep 2:233-251.

Foulkes D (1985) Dreaming: a cognitive-psychological analysis. Hillsdale, NJ: Lawrence Erlbaum.

Fox KC, Nijeboer S, Solomonova E, Domhoff GW, Christoff K (2013) Dreaming as mind wandering: evidence from functional neuroimaging and first-person content reports. Front Hum Neurosci 7:412.

Fredrickson BL (2013) Positive emotions broaden and build. In: Advances in experimental social psychology, Vol 47 (Devine G, Plant EA, eds), pp 1-53. San Diego: Academic.

Gable PA, Mechin NC, Hicks JA, Adams DL (2015) Supervisory control system and frontal asymmetry: neurophysiological traits of emotionbased impulsivity. Soc Cogn Affect Neurosci 10:1310-1315.

Gable PA, Neal LB, Threadgill AH (2018) Regulatory behaviour and frontal activity: considering the role of revised-BIS in relative right frontal asymmetry. Psychophysiology 55:e12910.

Gianotti LR, Knoch D, Faber PL, Lehmann D, Pascual-Marqui RD, Diezi C, Schoch C, Eisenegger C, Fehr E (2009) Tonic activity level in the right prefrontal cortex predicts individuals' risk taking. Psychol Sci 20:33-38.

Grimshaw GM, Carmel D (2014) An asymmetric inhibition model of hemispheric differences in emotional processing. Front Psychol 5:489.

Hagemann D, Naumann E, Thayer JF, Bartussek D (2002) Does resting electroencephalograph asymmetry reflect a trait? An application of latent state-trait theory. J Pers Soc Psychol 82:619-641.

Harmon-Jones E, Allen JJ (1998) Anger and prefrontal brain activity: EEG asymmetry consistent with approach motivation despite negative affective valence. J Pers Soc Psychol 74:1310-1316.

Harmon-Jones E, Gable PA (2018) On the role of asymmetric frontal cortical activity in approach and withdrawal motivation: an updated review of the evidence. Psychophysiology 55:e12879.

Harmon-Jones E, Sigelman J (2001) State anger and prefrontal brain activity: evidence that insult-related relative left prefrontal activation is associated with experienced anger and aggression. J Pers Soc Psychol 80: 797-803.

Hewig J, Hagemann D, Seifert J, Naumann E, Bartussek D (2004) On the selective relation of frontal cortical asymmetry and anger-out versus anger-control. J Pers Soc Psychol 87:926-939.

Hobson JA, Friston KJ (2012) Waking and dreaming consciousness: neurobiological and functional considerations. Behav Brain Sci 98:82-98.

Hobson JA, Pace-Schott EF, Stickgold R (2000) Dreaming and the brain: toward a cognitive neuroscience of conscious states. Behav Brain Sci 23: 793-842.

Horikawa T, Tamaki M, Miyawaki Y, Kamitani Y (2013) Neural decoding of visual imagery during sleep. Science 340:639-642.

Iber C, Ancoli-Israel S, Chesson A, Quan SF (2007) The AASM manual for the scoring of sleep and associated events: rules, terminology and technical specifications, Ed 1. Westchester, IL: American Academy of Sleep Medicine.

Jensen O, Mazaheri A (2010) Shaping functional architecture by oscillatory alpha activity: gating by inhibition. Front Hum Neurosci 4:186.
Jensen-Campbell LA, Knack JM, Waldrip AM, Campbell SD (2007) Do big five personality traits associated with self-control influence the regulation of anger and aggression? J Res Pers 41:403-424.

Kayser J, Tenke CE (2006a) Principal components analysis of Laplacian waveforms as a generic method for identifying ERP generator patterns: II. Adequacy of low-density estimate. Clin Neurophysiol 117:369-380.

Kayser J, Tenke CE (2006b) Principal components analysis of Laplacian waveforms as a generic method for identifying ERP genera-tor patterns: I. Evaluation with auditory oddball tasks. Clin Neurophysiol 117:348-368.

Kayser J, Tenke CE (2015) Issues and considerations for using the scalp surface Laplacian in EEG/ERP research: a tutorial review. Int J Psychophysiol 97:189-209.

Kelley NJ, Hortensius R, Schutter DJ, Harmon-Jones E (2017) The relationship of approach/avoidance motivation and asymmetric frontal cortical activity: a review of studies manipulating frontal asymmetry. Int $J$ Psychophysiol 119:19-30.

Kim SH, Hamann S (2007) Neural correlates of positive and negative emotion regulation. J Cogn Neurosci 19:776-798.

Klimesch W (2012) Alpha-band oscillations, attention, and controlled access to stored information. Trends Cogn Sci 16:606-617.

Klimesch W, Sauseng P, Hanslmayr S (2007) EEG alpha oscillations: the inhibition-timing hypothesis. Brain Res Rev 53:63-88.

Levin R, Nielsen TA (2007) Disturbed dreaming, posttraumatic stress disorder, and affect distress: a review and neurocognitive model. Psychol Bull 133:482-528.

Maquet P, Péters J, Aerts J, Delfiore G, Degueldre C, Luxen A, Franck G (1996) Functional neuroanatomy of human rapid-eye-movement sleep and dreaming. Nature 383:163-166.

Neal LB, Gable PA (2016) Neurophysiological markers of multiple facets of impulsivity. Biol Psychol 115:64-68.

Neal LB, Gable PA (2017) Regulatory control and impulsivity relate to resting frontal activity. Soc Cogn Affect Neurosci 12:1377-1383.

Nielsen TA (2010) Dream analysis and classification: the reality simulation perspective. In: Principles and practice of sleep medicine, Ed 5 (Kryeger M, Roth T, Dement WC, eds), pp 595-603. New York: Elsevier.

Nielsen TA, Chénier V (1999) Variations in EEG coherence as an index of the affective content of dreams from REM sleep: relationships with face imagery. Brain Cogn 41:200-212.

Nielsen T, Levin R (2007) Nightmares: a new neurocognitive model. Sleep Med Rev 11:295-310.

Nofzinger EA, Mintun MA, Wiseman M, Kupfer DJ, Moore RY (1997) Forebrain activation in REM sleep: an FDG PET study. Brain Res 770: 192-201.

Ochsner KN, Ray RD, Cooper JC, Robertson ER, Chopra S, Gabrieli JD, Gross JJ (2004) For better or for worse: neural systems supporting the cognitive down- and up-regulation of negative emotion. Neuroimage 23:483499.

Ochsner NK, Silvers JA, Buhle JT (2012) Functional imaging studies of emotion regulation: a synthetic review and evolving model of the cognitive control of emotion. Ann N Y Acad Sci 1251:E1-E24.

Perogamvros L, Schwartz S (2012) The roles of the reward system in sleep and dreaming. Neurosci Biobehav Rev 36:1934-1951.

Perogamvros L, Baird B, Seibold M, Riedner B, Boly M, Tononi G (2017) The phenomenal contents and neural correlates of spontaneous thoughts across wakefulness, NREM sleep, and REM sleep. J Cogn Neurosci 29: 1766-1777.

Perrin F, Pernier J, Bertrand O, Echallier JF (1989) Spherical splines for scalp potential and current density mapping. Electroencephalogr Clin Neurophysiol 72:184-187.

Rechtschaffen A, Kales A (1968) A manual of standardized terminology, techniques and scoring system for sleep stages of human subjects. Washington, DC: Government Printing Office.

Revonsuo A (2000) The reinterpretation of dreams: an evolutionary hypothesis of the function of dreaming. Behav Brain Sci 23:877-901.

Revonsuo A, Tuominen J, Valli K (2016) The simulation theories of dreaming: how to make theoretical progress in dream science. In: Open MIND: philosophy and the mind sciences in the 21st century (Metzinger $\mathrm{T}$, Windt JM, eds), pp 1341-1348. Cambridge, MA: Massachusetts Institute of Technology.

Reznik JS, Allen JJ (2018) Frontal asymmetry as a mediator and moderator of emotion: an updated review. Psychophysiology 55:e12965.

Salas CE, Castro O, Yuen KS, Radovic D, d'Avossa G, Turnbull OH (2016) 
'Just can't hide it': a behavioural and lesion study on emotional response modulation after right prefrontal damage. Soc Cogn Affect Neurosci 11:1528-1540.

Santesso DL, Segalowitz SJ, Ashbaugh AR, Antony MM, McCabe RE, Schmidt LA (2008) Frontal EEG asymmetry and sensation seeking in young adults. Biol Psychol 78:164-172.

Scarpelli S, D’Atri A, Gorgoni M, Ferrara M, De Gennaro L (2015a) EEG oscillations during sleep and dream recall: state or trait-like individual differences. Front Psychol 6:605.

Scarpelli S, Marzano C, D’Atri A, Gorgoni M, Ferrara M, De Gennaro L (2015b) State- or trait-like individual differences in dream recall: preliminary findings from a within-subjects study of multiple nap REM sleep awakenings. Front Psychol 6:928.

Schmidt LA, Cote KA, Santesso DL, Milner CE (2003) Frontal electroencephalogram alpha asymmetry during sleep: stability and its relation to affective style. Emotion 3:401-407.

Schredl M (2003) Continuity between waking and dreaming: a proposal for a mathematical model. Sleep Hypnosis 5:38-52.

Schredl M, Doll E (1998) Emotions in diary dreams. Conscious Cogn 7:634-646.

Schutter DJ, Harmon-Jones E (2013) The corpus callosum: a commissural road to anger and aggression. Neurosci Biobehav Rev 37:2481-2488.

Shapiro SS, Wilk MB (1965) An analysis of variance test for normality (complete samples). Biometrika 52:591-611.

Siclari F, Baird B, Perogamvros L, Bernardi G, LaRocque JJ, Riedner B, Boly M, Postle BR, Tononi G (2017) The neural correlates of dreaming. Nat Neurosci 20:872-878.

Sikka P, Valli K, Virta T, Revonsuo A (2014) I know how you felt last night, or do I? Self- and external ratings of emotions in REM sleep dreams. Conscious Cogn 25:51-66.

Sikka P, Feilhauer D, Valli K, Revonsuo A (2017) How you measure is what you get: differences in self- and external ratings of emotional experiences in home dreams. Am J Psychol 130:367-384.

Sikka P, Revonsuo A, Sandman N, Tuominen J, Valli K (2018) Dream emo- tions: a comparison of home dream reports with laboratory early and late REM dream reports. J Sleep Res 27:206-214.

Skancke FJ, Holsen I, Schredl M (2014) Continuity between waking life and dreams of psychiatric patients: a review and discussion of the implications for dream research. Int J Dream Res 7:39-53.

Smith EE, Reznik SJ, Stewart JL, Allen JJ (2017) Assessing and conceptualizing frontal EEG asymmetry: an updated primer on recording, processing, analysing, and interpreting frontal alpha asymmetry. Int J Psychophysiol 111:98-114.

Spoormaker VI, Montgomery P (2008) Disturbed sleep in post-traumatic stress disorder: secondary symptom or core feature? Sleep Med Rev 12:169-184.

Sterpenich V, Perogamvros L, Tononi G, Schwartz S (2019) Fear in dreams and in wakefulness: evidence for day/night affective homeostasis. bioRxiv. Available at https://doi.org/10.1101/534099.

Tomarken AJ, Davidson RJ, Wheeler RE, Doss RC (1992) Individual differences in anterior brain asymmetry and fundamental dimensions of emotion. J Pers Soc Psychol 62:676-687.

Vijayan S, Lepage KQ, Kopell NJ, Cash SS (2017) Frontal beta-theta network during REM sleep. Elife 6:e18894.

Wamsley EJ (2013) Dreaming, waking conscious experience, and the resting brain: report of subjective experience as a tool in the cognitive neuroscience. Front Psychol 4:637.

Wamsley EJ, Stickgold R (2011) Memory, sleep and dreaming: experiencing consolidation. Sleep Med Clin 6:97-108.

Wehrle R, Kaufmann C, Wetter TC, Holsboer F, Auer DP, Pollmächer T, Czisch M (2007) Functional microstates within human REM sleep: first evidence from fMRI of a thalamocortical network specific for phasic REM periods. Eur J Neurosci 25:863-871.

Windt JM (2015) Dreaming: a conceptual framework for philosophy of mind and empirical research. Cambridge, MA: Massachusetts Institute of Technology.

Winkler I, Haufe S, Tangermann M (2011) Automatic classification of artifactual ICA-components for artefact removal in EEG signals. Behav Brain Funct 7:30. 